\title{
The concept of 3D ECT system with increased border area sensitivity for crystallization processes diagnosis
}

\author{
Radostaw Wajman \\ Institute of Applied Computer Science, Lodz University of Technology, Lodz, Poland
}

\begin{abstract}
Purpose - Crystallization is the process widely used for components separation and solids purification. The systems for crystallization process evaluation applied so far, involve numerous non-invasive tomographic measurement techniques which suffers from some reported problems. The purpose of this paper is to show the abilities of three-dimensional Electrical Capacitance Tomography (3D ECT) in the context of non-invasive and non-intrusive visualization of crystallization processes. Multiple aspects and problems of ECT imaging, as well as the computer model design to work with the high relative permittivity liquids, have been pointed out.

Design/methodology/approach - To design the most efficient (from a mechanical and electrical point of view) 3D ECT sensor structure, the highprecise impedance meter was applied. The three types of sensor were designed, built, and tested. To meet the new concept requirements, the dedicated ECT device has been constructed.

Findings - It has been shown that the ECT technique can be applied to the diagnosis of crystallization. The crystals distribution can be identified using this technique. The achieved measurement resolution allows detecting the localization of crystals. The usage of stabilized electrodes improves the sensitivity of the sensor and provides the images better suitable for further analysis.

Originality/value - The dedicated 3D ECT sensor construction has been proposed to increase its sensitivity in the border area, where the crystals grow. Regarding this feature, some new algorithms for the potential field distribution and the sensitivity matrix calculation have been developed. The adaptation of the iterative 3D image reconstruction process has also been described.
\end{abstract}

Keywords Crystallization, Image reconstruction, 3D ECT, Electric field modeling, Non-invasive imaging

Paper type Research paper

\section{Introduction}

Crystallization is the process by which a solution component goes from liquid to solid in a frame of crystals. It is widely used as an industrial method for components separation ( $\mathrm{Li}$ et al., 2004; Mullin, 2001). It is because of its additional advantage that apart of separation offers solids purification. Nowadays, when the industry touches the high pressure on optimization of energy and time consumption in parallel to the high production quality, the crystallization process, known for over 100 years, can meet these requirements. It is an integral part of productions' lines in many areas of industry as food production (sucrose) (Rózsa, 1996; Rózsa et al., 2016; Temerk et al., 2014; Villanueva et al., 2015), pharmacy (drugs) (Besenhard et al., 2014; Gao et al., 2017; Shekunov and York, 2000) but also in chemical flows (limescale in pipelines) (Butler et al., 2006; Georgiou et al., 2018), nanoparticles and polymerization (Hierzenberger et al., 2010; Ohta et al., 2005). That is why many research teams are dealing with the task of developing new methods for diagnosis, modeling, and control of

The current issue and full text archive of this journal is available on Emerald Insight at: https://www.emerald.com/insight/0260-2288.htm

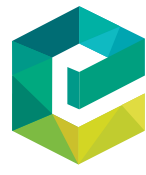

Sensor Review

41/1 (2021) 35-45

Emerald Publishing Limited [ISSN 0260-2288]

[DOI 10.1108/SR-10-2019-0254] crystallization processes (Fujiwara et al., 2005; Gao et al., 2017; Jha et al., 2017; Winn and Doherty, 2000).

Efficient process control ensures its correct performance. This, in turn, has an impact on crystals' purity, size, shape and distribution (Winn and Doherty, 2000). Besides these mentioned, for the crystallization process assessment, other

(C) Radosław Wajman. Published by Emerald Publishing Limited. This article is published under the Creative Commons Attribution (CC BY 4.0) licence. Anyone may reproduce, distribute, translate and create derivative works of this article (for both commercial \& non-commercial purposes), subject to full attribution to the original publication and authors. The full terms of this licence may be seen at http://creativecommons.org/licences/ by $/ 4.0 /$ legalcode

The author wants to thank Dr Mariusz Rzasa, Prof. Dominik Sankowski, Dr Robert Banasiak, Dr Tomasz Jaworski and Dr Jacek Nowakowski for their help and fruitful advice.

This work was financed by the Lodz University of Technology, Faculty of Electrical, Electronic, Computer and Control Engineering as a part of the Polish National Centre for Research and Development - project No. POIR.04.01.02-00-0089/17-00.

Funding: National Centre for Research and Development Fundator in polish: Narodowe Centrum Badań i Rozwoju Project: POIR.04.01.02-000089/17-00.

Received 28 October 2019

Revised 2 January 2020

17 March 2020

2 October 2020

Accepted 14 October 2020 
properties are also defined as the rate of crystals growth and nucleation, a polymorphic form of the crystals. Understanding all of them allows maintaining the process to be efficient and obtaining high quality of the product.

The aspects described so far concern mostly the diagnostic part. However, the efficiency of the process depends also on the reactors (crystallizers) design and manufacturing. Many problems are associated with this issue as described in (Barrett et al., 2005; Correa et al., 2012). The designing stage should be supported with modeling of the crystallization phenomenon. For this task, the methods based on the inverse problem for population balance equations (Barrett et al., 2005; Correa et al., 2012; Villanueva et al., 2015) or the saturation variance in images (Kawabata, 2011) are commonly applied. The solution also allows developing the model-based algorithm for control, which would be helpful in the prediction of the facility and working conditions.

Unfortunately, many industrial solutions still involve the visual assessment of the technicians (i.e. a process expert's observation) and their response to the adverse states. This treatment would not work in case of quick thermodynamic, kinetic changes, sudden pollutions or neglectful mixing etc. Then, it seems to be natural to work out an accurate, relatively inexpensive and either non-invasive, non-intrusive diagnostic system.

The systems for crystallization process evaluation, applied so far, involve numerous tomographic measurement techniques using various physical properties as X-rays, optics, ultrasound and finally electric and magnetic field. Some examples of Computed X-ray Tomography (CT) applications can be found in (Bischof et al., 2007; Carlson and Denison, 1992). In the case of both, the authors proved the high abilities for crystals detection. Nevertheless, they mentioned some problems, especially with the inhomogeneous sensitivity of the measurements in the different areas. It must also be noted that the usage of $\mathrm{X}$-rays meets the high requirements for hard-field radiation relating to working security and building adaptation.

Relatively less demanding is the optical tomography (Abbas et al., 2002), which is also suitable for the crystals size evaluation. Similarly, in the case of the research described in (Hierzenberger et al., 2010), the Optical Coherence Tomography was successfully applied to identify polymer crystallization. The measurement resolution in the range of micrometers was obtained thanks to the optical dispersing medium, the light beam of wavelength close to infrared and the waves interference phenomenon. Therefore, the multilayer crystals growth detection was possible according to the depth scanning and time-changing registration. The disadvantage of the optical-based techniques is a necessity of building transparent tanks. This condition, unluckily, is difficult to meet especially in the case of crystals growing on the wall's surface.

The measurement techniques based on ultrasound or electric phenomena are free from this restriction. The authors of (Mougin et al., 2003) built the Ultrasound Spectroscopy system and obtained a reasonable sensitivity of measurements. Unfortunately, they observed undesirable crystals breakage. Next, the application of the electrical impedance spectroscopy in the crystallization process diagnosis has been presented in (Zhao and Hammond, 2011). In this case in turn, the authors pointed out the negative impact of changing process conditions on the measurement stability. It was observed the significant phase angle shift regarding the changing process temperature, which is crucial in crystals growth rate control.

Concerning the tomography based on the electric field, mostly two properties are measured: conductivity and permittivity. In a frame of the work described in (Primrose, 2008), the Electrical Resistance Tomography (ERT) was used to measure the conductivity distribution of the solution under different conditions. It can be read that ERT is an appropriate technique to evaluate the saturation level within the different regions. Unfortunately, the authors did not discuss the noticeable problem of crystals' growth on the resistance electrodes surface. There is a high risk of losing the connection between the thin electrodes and the solution that is the basis of ERT.

The authors of (Harada et al., 2018; Ohta et al., 2005) proposed the capacitance measurement system based on the electric circuit that involves the RLC meter and only one pair of electrodes. Such a strategy provides a simple information in the form of IS or NOT. It does not meet the requirements of capacitance tomography conception, where the set of electrodes is located around the process. Then, the sequence of capacitance measurements between all the possible electrode pairs can be collected. Next, applying sophisticated algorithms, the image of permittivity distribution in the process interior can be reconstructed. The image, finally, corresponds to the density of the components.

The problems listed above, regarding the developed tools for the crystallization process identification, suffer from different disadvantages. Some of them offer the diagnostic signal with significant measurement uncertainty, i.e. the general presence of crystals but without any details about them. There are methods, in turn, which provides a precise knowledge about the process, but the application requirements are too challenging to meet in the industrial conditions. Basically, in the state-of-theart, the application of 3D Electrical Capacitance Tomography (ECT) in the context of crystallization process diagnosis cannot be found. Hence, this study will show the ability of ECT.

Table 1 summarizes the applied so far diagnostics systems for crystallization purposes.

\section{Three-dimensional electrical capacitance tomography (3 D ECT)}

ECT (Kryszyn et al., 2014, 2017; Rashid et al., 2016) is a noninvasive measurement technique and regarding the mentioned ERT constraints does not require continuous electric contact with the solution. Depends on the measurement concept and the arrangement of the electrodes, ECT can provide 2D (cross-section) or 3D imaging of the process interior (Kowalska et al., 2019; Mazurkiewicz et al., 2005; Rymarczyk, 2016; Smolik et al., 2017; Soleimani, 2006; Wang et al., 2010). So far, there are many successful applications of ECT for numerous industrial processes monitoring as two-phase gas-liquid flows (Fiderek et al., 2017; Rzasa, 2009), solids pneumatic conveying (Romanowski et al., 2017), silo discharging (Garbaa et al., 2016; Grudzien et al., 2010, 2012), flames (Wang et al., 2010) and concrete beams studies (Grudzien et al., 2016) and many others.

In this study, solely the $3 \mathrm{D}$ case has been considered. In this form, the ECT can reflect the spatial nature of the process in a more reliable way. One needs to be noted. The 3D imaging is more demanding from the computational power point of view, 
Table 1 Summary of measurement systems applied so far for crystals growth diagnosis

\begin{tabular}{|c|c|c|c|}
\hline $\begin{array}{l}\text { Diagnosis method } \\
\text { (reference) }\end{array}$ & $\begin{array}{l}\text { Accuracy, } \\
\text { measurement resolution }\end{array}$ & Measurement frequency & Comments \\
\hline $\begin{array}{l}\text { X-ray tomography } \\
\text { (Bischof et al., 2007; } \\
\text { Carlson and Denison, 1992) }\end{array}$ & $\begin{array}{l}\text { high abilities of detection, } \\
\text { high resolution }\end{array}$ & 60-180 min/image & $\begin{array}{l}\text { inhomogeneous sensitivity, } \\
\text { - } \begin{array}{l}\text { hard-field radiation } \\
\text { requirements }\end{array}\end{array}$ \\
\hline $\begin{array}{l}\text { Optical tomography } \\
\text { (Abbas et al., 2002) }\end{array}$ & high resolution & few frames/ second & $\begin{array}{l}\text { - transparent medium and tank } \\
\text { required }\end{array}$ \\
\hline $\begin{array}{l}\text { Optical Coherence } \\
\text { Tomography } \\
\text { (Hierzenberger et al., 2010) }\end{array}$ & $\begin{array}{l}\text { high resolution of several } \\
\text { hundred microns }\end{array}$ & few frames/ second & $\begin{array}{l}\text { - partially transparent medium } \\
\text { acceptable, } \\
\text { - transparent tank required }\end{array}$ \\
\hline $\begin{array}{l}\text { Ultrasound spectroscopy } \\
\text { (Mougin et al., 2003) }\end{array}$ & $\begin{array}{l}\text { high resolution (single crystals } \\
\text { detection) }\end{array}$ & few frames/ second & $\begin{array}{l}\text { - invasive for (L) -Glutamic Acid } \\
\text { Crystals growth }\end{array}$ \\
\hline $\begin{array}{l}\text { Electrical Resistance } \\
\text { Tomography (Primrose, } \\
\text { 2008) }\end{array}$ & $\begin{array}{l}\text { low resolution (crystals } \\
\text { regions' detection) }\end{array}$ & $\begin{array}{l}\text { up to } 100 \text { frames per second } \\
\text { for } 12 \text { electrodes sensors }\end{array}$ & $\begin{array}{l}\text { - connection with the process, } \\
\text { crystals growth on electrodes, } \\
\text { high conductivity medium, } \\
\text { - possible 3D imaging }\end{array}$ \\
\hline $\begin{array}{l}\text { Electrical Capacitance } \\
\text { measurement (Al Hosani } \\
\text { et al., 2015) }\end{array}$ & $\begin{array}{l}\text { - low resolution, } \\
\text { - crystals regions' } \\
\text { detection, } \\
\text { for limescale } \\
\text { detection }\end{array}$ & $\begin{array}{l}\text { up to } 100 \text { frames per second } \\
\text { for } 12 \text { electrodes sensors }\end{array}$ & $\begin{array}{l}\text { no contact with a medium, } \\
\text { - low medium conductivity } \\
\text { recommended, } \\
\text { possible 3 D imaging }\end{array}$ \\
\hline
\end{tabular}

because of the significantly bigger size of the reconstructed images. This has an impact on the sensitivity matrix complexity used for both reconstruction algorithms and Finite Elements Method (FEM) calculation (Rymarczyk, Tchorzewski and Sikora, 2017). To consider the 3D ECT imaging as a real-time (on-line) diagnostic system saving the reasonable image quality at the same time, the massive-parallel computation GPU techniques were involved (Banasiak et al., 2014; Kapusta et al., 2012).

Some flaws of ECT measurement of liquids with a high value of relative electrical permittivity

The most common ECT sensor design considers the electrodes mounted on the outer side of the pipeline (here reactor). From the crystallization point of view, this is quite a favorable approach that protects the surface of the electrode from the process. Unfortunately, in the case of liquid with a high value of relative electrical permittivity distribution, as sucrose solution, the following problem may be noticed. The electric field insufficiently penetrates the examined process. This phenomenon will be explained in detail within the next section, together with the FEM simulation results. Therefore, undesirable inter-electrodes measurement values can be obtained. For the neighboring electrodes and the sensor filled with the solution, the measured capacitance value can be lower than the same measurement for a sensor with air.

To explain the problem, the sequence of the three following measurement vectors is presented in Figure 1. The measured capacitances, obtained for the sensor filled with the sucrose solution, were normalized according to the procedure described e.g. in (Loser et al., 2001) - eq. (1). All the measured values, in this case, should be very close to 1 . Unfortunately, for some of the neighbored pairs of electrodes, the significant
Figure 1 Instabilities of normalized measured capacitance values for ECT sensor filled with sucrose solution

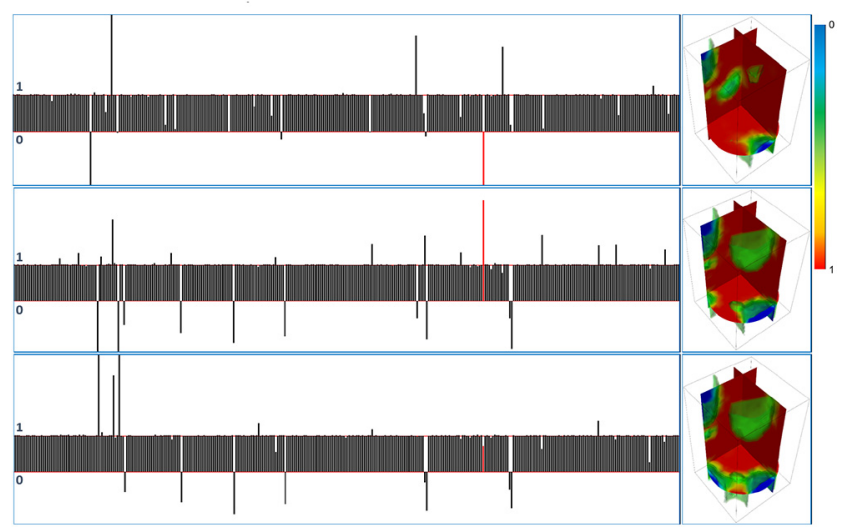

instability can be observed. Let's consider one measurement value highlighted in red. In the first set, its value is lower than 1 . In the next, it is greater than 1 , but in the last, it is in range 0-1. On the right side of the measurement vectors, the reconstructed $3 \mathrm{D}$ images of normalized permittivity distributions are presented. The red color and value 1 mean the sucrose solutions, blue color and 0 correspond with sucrose crystals concentration. The images reflect the instabilities in the form of chaotic artifacts instead of the homogenous solution. It is also caused by the arduous way of emptying the wet sensor for the normalization procedure. Anytime, some drops of the solution remain on the reactor's wall. The drops are random and have a significant impact on the reference measurement vector being 
processed in normalization. In the laboratory set-up, it is possible to dry the wall, but in the industrial conditions, this may be a severe obstacle.

Within the next sections, the ECT sensor with the electrodes mounted on the inner sensor side will be considered. Additionally, this study involves the conception of so-called stabilizing electrodes used for the first time in (Rzasa et al., 2007) only in 2D case for the counter-current gas-liquid flow monitoring. The designed electrodes structure caused a sensitivity increase in the border area of the sensor. In the case of the crystallization process, it is essential to have stabilization electrodes because the crystals mostly grow on the sensor's wall. The obtained results of experiments presented in this article show that the application of stabilization electrodes allows getting a higher precision in crystallization ECT diagnosis.

\section{Research setup}

This study considers the applicability of the 3D ECT technique to monitor the distribution of the crystals. To design the most efficient (from a mechanical and electrical point of view) sensor structure, the high-precise impedance meter (Agilent E4980A) was applied. The ECT sensor properties (i.e. electrodes layout, their shapes and geometrical dimensions, horizontal and vertical gaps, the width of the inner-electrodes rings, boundary screens and finally the thickness of the isolation) were determined according to the measuring sensitivity abilities of the hardware unit and to the dielectric properties of the sucrose solution. The beet juice has a relative electrical permittivity value higher than e.g. tap water.

The following three types of sensor construction were designed, built and tested (see models depicted in Figure 2). Each sensor consists of 32 electrodes located around the reactor in 4 layers. Below and above the electrodes' layers, the axial shields are designed, which, together with the radial shield surrounding the sensor, protect against the outer electrostatic field. The radial shield is distanced from the sensor by the polyurethane (PU) foam with a width of $1 \mathrm{~cm}$. The model (a) has the capacitance electrodes mounted outside the reactor's PVC wall. In the next (b) model in Figure 2, the electrodes are mounted on the inner side of the reactor's wall. Similarly, in the model (c) the electrodes are inside the reactor, but also between the measuring electrodes, the set of thin electrodes can be seen. These thin electrodes are called stabilizing electrodes.

In the case of models (a) and (b), the set of capacitances $C_{x}$ is measured between all possible electrodes pairs including electrodes from different layers. During the single measurement, the positive potential $V$ is set on one electrode (called as excited) and the rest are grounded. Now, the capacitances of all electrodes being in pair with the excited one can be measured. Then, the next electrode can be considered as excited. For one excited electrode, one simulation with different boundary conditions needs to be performed. The measurement strategy in the model (c) considers 64 electrodes in total (32 measuring and 32 stabilizing). Here, during the single measurement (for instance, $1-5$ where the $1^{\text {st }}$ is excited) the 10 times lower potential value, then $V$, is set only on two stabilizing electrodes neighboring with the measuring (here the $5^{\text {th }}$ ) electrode. The rest of them are grounded. For the next electrodes pair (i.e. 1-6) the different stabilizing electrodes get the potential. As it can be seen for one excited electrode 31 simulations with the different boundary conditions need to be solved.

The electrical field inside an ECT sensor can be simulated with the usage of the Laplace's equation (assuming no source charge) (Sikora, 2007):

$$
\nabla^{2} \phi=\nabla \cdot(\varepsilon(\vec{x}) \nabla \phi(\vec{x}, t))=0
$$

where $\nabla^{2}$ is the Laplace's operator, $\phi(\vec{x}, t)$ is the electrical potential, and $\varepsilon(\vec{x})$ is the electrical permittivity. The vectors $\vec{x}$ are the coordinates in the sensor volume. The ECT measurement unit applied during the experiments generates an alternating current at the fixed frequency. If the potential field $\phi(\vec{x})$ is known, the induced charge $Q_{R}$ at the receiver electrode can be determined using the Gauss's law:

$$
Q_{R}=\oiint_{S_{R}} \varepsilon \vec{E} d \vec{S}
$$

where $S$ is the area of the electrode and $\vec{E}$ is the electric field. In the case of the model (c) the capacitance vector will be the sum of the results from the three different simulations with different boundary conditions. The boundary condition on the excited electrode is defined as $\varphi=V$ and next on the thin stabilizing electrodes near the receiver as $\varphi=V / 10$. Only those mentioned electrodes are held with a positive voltage. The others stay grounded. The result capacitance is then given as:

$$
C_{X}=\frac{Q_{X p R}}{U_{X p R}}+\frac{Q_{X 1 R}}{U_{X 1 R}}+\frac{Q_{X 2 R}}{U_{X 2 R}}=C_{X P}+C_{X 1}+C_{X 2}
$$

The voltages $U_{X p R}, U_{X 1 R}$ and $U_{X 2 R}$ are the electrical potentials between the receiver electrode $\mathrm{R}$ and the excited electrode $\mathrm{Xp}$ and thin electrodes $\mathrm{X} 1$ and $X 2$ respectively (see electrodes' symbols in Figure 2(c) with blue labels) where the following charges $Q_{X p R}, Q_{X 1 R}, Q_{X 2 R}$ were induced.

To meet the new concept requirements, the dedicated ECT device has been constructed, which can freely manage the strategy of electrodes' potentials definition. The measurement complex system [presented in Figure 3(b)] consists of the mentioned RLC meter and the box with a set of configurable relays managed by RPi3 module. The dedicated software has been implemented to manage the measurement procedure.

In Figure 3(a) the research facility is presented. It consists of the batch-reactor to study the crystallization process and the remote GUI-based software system to monitor and control the reactor's functioning. The logic of the control module works in a cloud as a back-end web-application providing for its clients a front-end GUI (mobile) control panel. The reactor with a diameter 14,2 cm and a high $20 \mathrm{~cm}$ ( $\sim 3 \mathrm{~L}$ of volume for sucrose solution) is equipped with the copper coil and with the motor stirrer. The copper coil is electrically connected with axial and radial shields and warms up the solution by the heating liquid. The following numbers listed in Figure 3(a) indicates (1) reactor, (2) control unit managed by RPi3 module, (3) GUIbased software of control panel, (4) boiler, (5) pump, (6) stirrer motor, (7) stirrer and (8) copper coil. Two thermo-couples have been connected to the control unit to measure and control the temperatures in the boiler and the reactor.

The crystallization is a long-term process. The stage of preparation of the saturated sucrose solution takes about 
Figure 2 Three types of ECT sensor modelsNotes: (a) with external electrodes, (b) with internal electrodes, (c) with additional thin stabilizing electrodes.

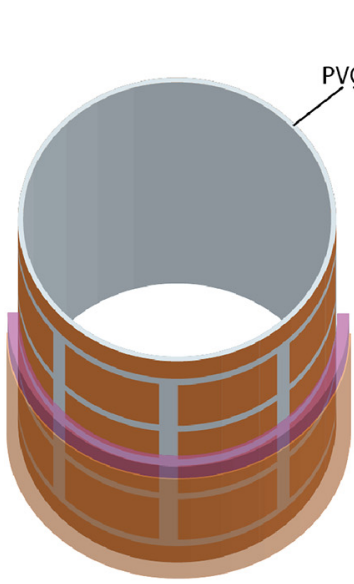

(a)

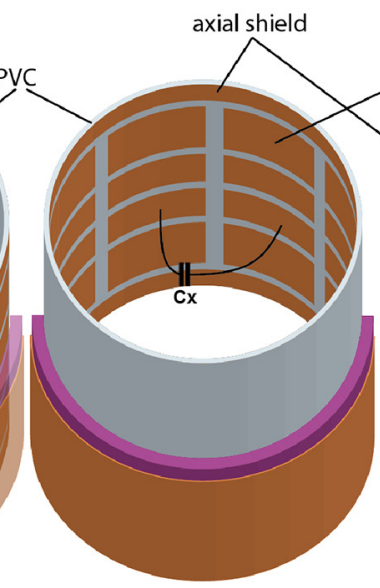

(b)

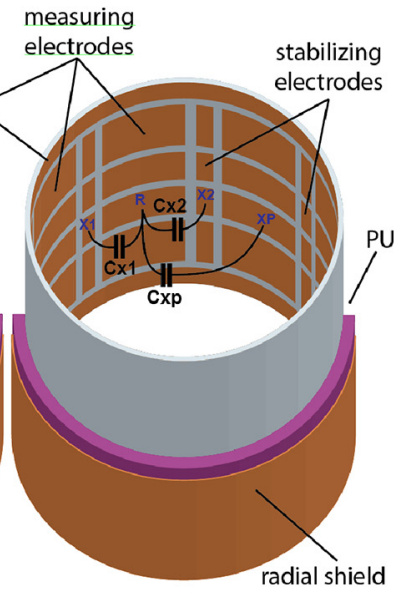

(c)

Notes: (a) With external electrodes; (b) with internal electrodes; (c) with additional thin stabilizing electrodes

Figure 3 Photos of the research facility and the multi-switching ECT measurement system

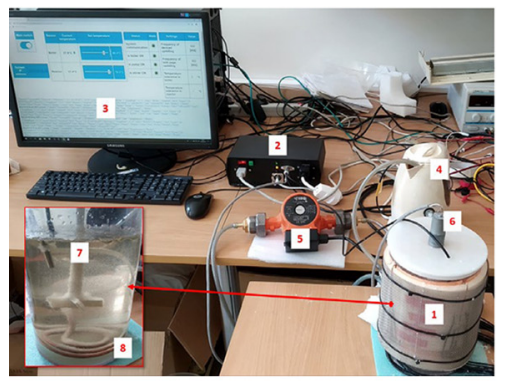

(a)

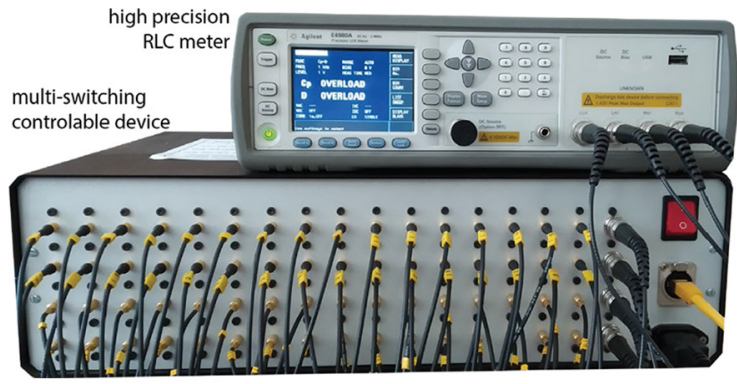

(b)
8 hours in the research facility. The sucrose portion of $320 \mathrm{~g}$ is dissolved in each $100 \mathrm{~mL}$ of water according to the solubility curve in the function of temperature given in (Rózsa, 1996; Temerk et al., 2014). Water is a good solvent for sucrose because there is a significant difference in its solubility depending on the temperature changes. Finally, the solution was warm up to $70^{\circ} \mathrm{C}$ and mixed until a total sucrose dissolution. Next, the stirrer was removed, and the capacitance normalization process was performed. Then, the solution was left for cooling down. Note, that in the industrial installations, the crystallization proceeds based on water evaporation in the temperature about $95^{\circ} \mathrm{C}$. It is much faster and efficient, but such conditions impose to use different high-temperatureresistant materials. In the laboratory, the crystals' growth on the basis of cooling was sufficient enough for this study.

The next day, on the reactor's wall and the solution's surface, the sucrose crystals were observed. The first photo (a) in Figure 4 presents the sensor with a stirrer (1), cover (2) and outer ECT sensor's screen (3). Next, the photo (b) presents the reactor interior with crystals (4) grown on the wall. The ECT measurements were continuously collected with a frequency of $1 \mathrm{~h}$ since the saturated solution was ready.

\section{Computer model and image reconstruction}

The mathematical basis of the electrostatic field phenomenon inside the ECT sensor as well as the principles of ECT image reconstruction process has been considered, so far, in many papers (Banasiak et al., 2010; Cui et al., 2016; Isaksen, 1996; Panczyk and Sikora, 2016; Rymarczyk, Tchorzewski, Adamkiewicz, et al., 2017; Yang and Peng, 2003). In this section, the differences in processing depending on the sensor model (see Figure 2) (i.e. electrodes layouts and measurement strategy) will be explained.

For purposes of the image reconstruction process, the author used his software for comprehensive processing and visualization of 3D electrical tomography data (Banasiak et al., 2017). Apart from the reconstruction algorithms, the software has implemented modules for mesh generation, electrodes layouts definition, electric field and capacitances calculation, sensor sensitivity distribution analysis, and finally tomography device connection and configuration.

The first step of the image reconstruction process is to build the computer model. It consists of the mesh of tetrahedrons (voxels) and the definition of electrodes layout (i.e. mapping of 
Figure 4 Working batch crystallization reactor with 3D ECT sensor (a) and the sucrose crystalized on the reactor's internal wall (b)

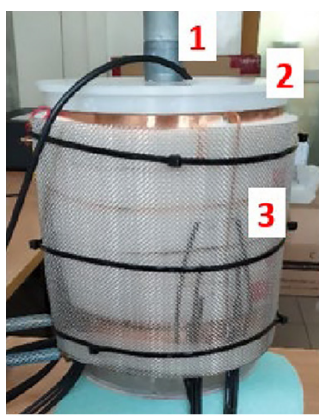

(a)

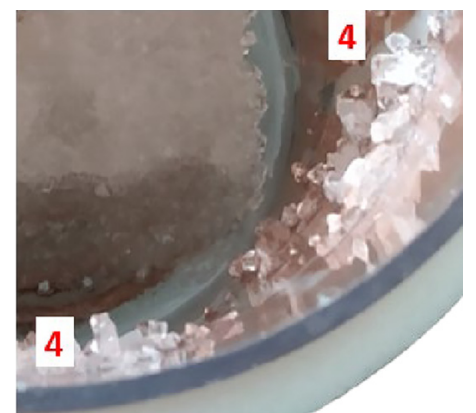

(b) electrodes surfaces onto triangular sides of tetrahedrons). The computer model is a fundamental part of two stages of iterative reconstruction: forward and inverse processing. The first one aims to calculate the electric field distribution and then to determine the sensor sensitivities distribution, which defines the influence of the permittivity change in a particular voxel on a change of measured capacitance. During the inversion, in turn, the image of spatial permittivity distribution is reconstructed in the voxels of the $3 \mathrm{D}$ mesh. The calculation of the electric field distribution may be completed by the implementation of the Finite Element Method and definition of first-order boundary conditions - Dirichlet (potentials values in nodes which correspond to the surfaces of the electrodes) and of the second-order - Neumann (permittivity values in voxels). However, to clearly show the differences between the three ECT sensors presented in Figure 2, the complete sensor model has been introduced, which considers not only the inner sensor volume but also the construction's elements as PVC pipe (reactor), PU foam and copper shields. In Figure 5 the three-dimensional mesh adopted to the computer model construction is depicted. On the left in the cross-sectional view of the mesh can be seen together with the distinction of PU, PVC materials. Mesh is constructed using growing rings starting from the sensor's center. In the middle, some details about their radius and elements density are listed. The elements density is given as a distance between two nodes on a ring. So, decreasing the value, the denser mesh is expected.
The mesh, in the part of inner sensor volume, is not homogenous as usually. It is denser in the border areas because the most changes in permittivity distribution (i.e. crystals) and also the rapid changes in the potentials' distribution (i.e. stabilizing electrodes in the model (c)) are expected right there.

The three different computer models for each sensor from Figure 2 have been created. The electric field (Figure 6) and sensitivity models (Figure 7) have been determined. The following images in Figure 6 show the normalized potentials values distributions and the equipotential lines. The visualization has been limited to the sensor interior including only the reactor wall and electrodes layout to explain some observed dependencies better. In the case of the model (a), where the electrodes were mounted outside the reactor wall, the insufficient penetration of the electric field into the examined process can be observed. When the electric field has on its path (between the excited and measuring electrodes) a closed area with a significantly higher permittivity value (as sucrose solution in contrast to the PVC reactor's wall), to reach the aim, it tries to find another way not to get across this area. The equipotential lines are mainly focused in the area of the reactor's wall (see the sample in the magnifier). This phenomenon mostly has an impact on the measurements for neighboring electrodes pairs, as shown in Figure 1. Model (b) considers the electrodes mounted inside the reactor. Here, by following the equipotential lines, the electric field is much more efficient to spread to the volume. However, in the case of the Model (c) where the additional stabilizing electrodes were involved, the results of the electric field calculation are most promising. The area of higher potentials values penetrates the volume center what goes hand in hand with the higher field energy in the border areas.

These considerations can also be observed while sensitivities distribution analysis. For the classical ECT sensor geometry and having the energy of the potential field, the elements of the sensitivity matrix can be calculated using the following equation (Yang and Peng, 2003):

$$
S_{i(X R) j}=-\int_{\gamma_{j}} \frac{E_{X, j}}{V_{X}} \frac{E_{R, j}}{V_{R}} d j
$$

where $E_{X, j}$ and $E_{R, j}$ are the values of the electric field in voxel $j$ when on the electrodes $\mathrm{X}$ (excited) and $\mathrm{R}$ (receiver) the

Figure 5 Three-dimensional mesh adopted to the computer models construction
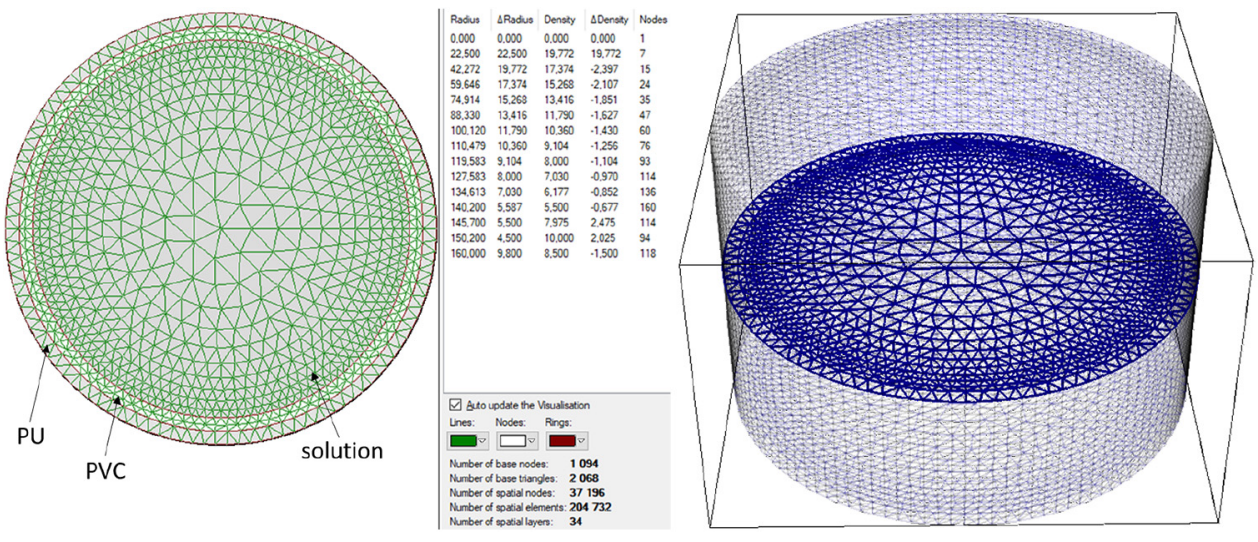
Figure 6 Images of normalized potentials distributions for three sensors models

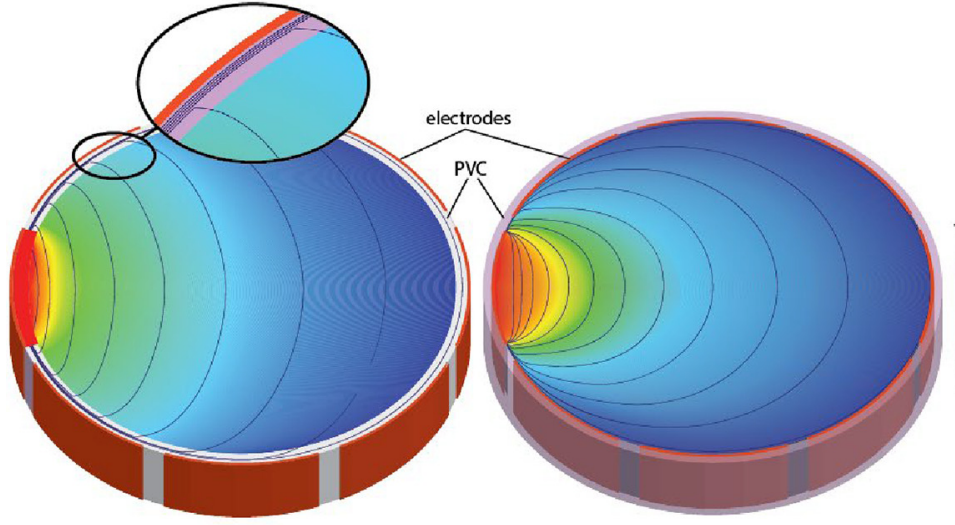

(a) (b)

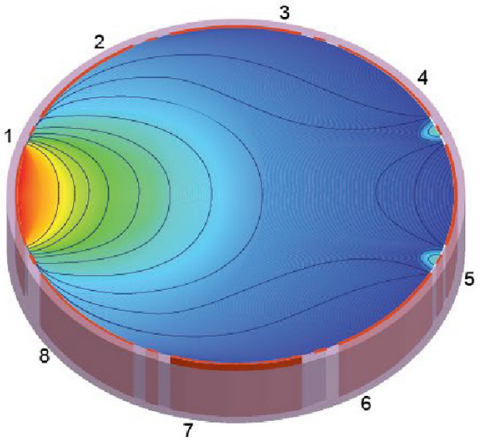

(c)
Figure 7 Normalized sensitivity maps for considered three sensors models

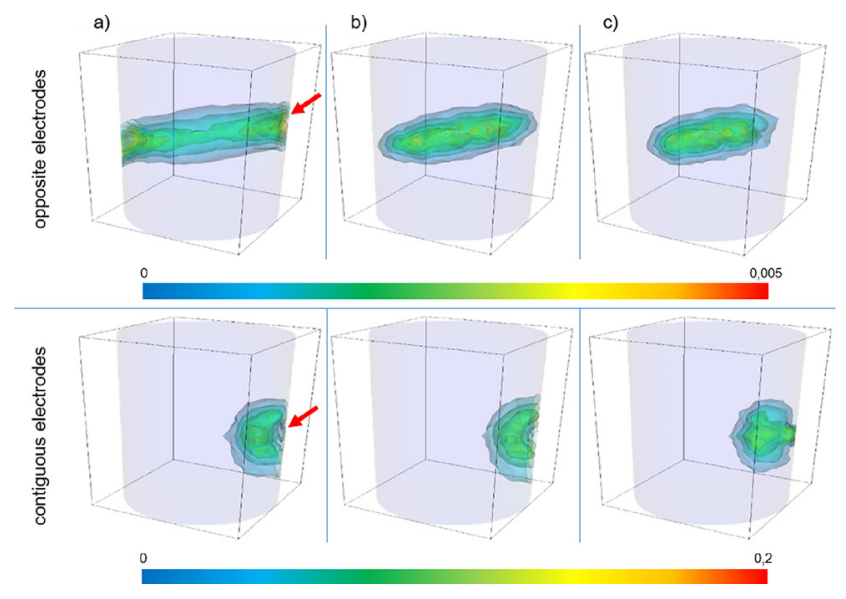

potentials $V_{X}$ and $V_{R}$ were set respectively, $\gamma_{j}$-is the volume of the $j$ voxel in the mesh. The considered electrodes' pair $X-R$ is the $i$ th measurement. In the next step, such sensitivity matrices should be determined for each electrode from the set (one measured and two stabilizing) according to equation (3). Then, as a result, the final sensitivity matrix is calculated as a sum of three components:

$$
S_{X}=S_{X 1}+S_{X 2}+S_{X p}
$$

where the indexes $\mathrm{X} 1, X 2$ and $\mathrm{Xp}$ have the same meaning as in the case of the equation (3).

For the three sensor models, the sensitivity matrices were determined, normalized, and depicted in Figure 7 as sensitivity maps for opposite and neighboring electrodes pairs.

In model (a) in the border areas, the increased normalized sensitivity values and rapid gradient can be noticed (especially next to the surfaces of the electrodes). This reflects the high gradient of potentials in these areas. The arrows point the location between neighboring electrodes where the most significant gradient of sensitivities occurred. It suffers from the random artifacts in images (see reconstructed images in Figure 1). It is difficult to distinguish them as crystals or not. Because the electric field more efficiently penetrates the process in models (b) and (c), the sensor is more sensitive to electrical permittivity changes between the center and border area. Additionally, in the model (c), the applied stabilizing electrodes induce the sensitivities gradient reduction just next to the surface of the electrode. It can be especially seen in the case of neighboring electrodes pair. The thin electrodes push the electric field into the reactor's volume.

\section{Results and discussion}

The research strategy considers three separate experiments with the three sensors (one by one) presented in Figure 2. Each sensor was mounted around the separate batch-reactor (photo (a) in Figure 4), then connected to the measurement unit [Figure 3(b)]. In each reactor, a similar crystallization process was conducted maintaining the same conditions i.e. the sucrose solution's composition, temperature, and time of heating and cooling. The ECT measurements were collected keeping the same time regimes and then finally normalized. The 3D image reconstruction of the normalized permittivity distribution was performed. The results are presented in Figure 8 and classified regarding the sensor model (a) - (c). Under the same reconstruction conditions (i.e. total iterations number 100, relaxation parameter 0.1 ), the truncated Landweber algorithm was applied in each case.

Only in models (b) and (c) the area of crystallized sucrose can be distinguished. Moreover, the model (c) allows determining the differences in crystals distribution. It is possible there to point the condensed areas [see arrows Figure 8 (c)].

Only in the case of the model (a) to obtain a reasonable image, one of the features of the multi-switching device was used. The measurements for neighboring electrodes pairs were neglected. This surely produced the image without artifacts but also induced the minor changes regarding the distribution of the crystals. Such an approach cannot be accepted in case of further image processing and analysis. 
The presented in Figure 8 images have been reconstructed from the tomographic measurements performed for the real crystallization process. Therefore, to analyze the images' quality, none of the standard criteria like the normalized mean square error or the Pearson's linear correlation coefficient (Yang and Peng, 2003) could be applied.

However, to provide the reader a tool for self-assessment of the 3D ECT images' quality and the usefulness of the new 3D ECT sensor's concept, the two charts with normalized values of phases density have been depicted in Figure 9. The values have been transferred from the reconstructed images for sensors (a), (b) and (c) (Figure 8). They have been scanned from the image's points located on a path determined between the centers of selected opposite electrodes pairs i.e. 9-13 and 19-23. The given electrodes pairs belong to the different electrodes layers i.e. are located on the different height of the reactor. The horizontal axis of the charts corresponds with the reactor's diameter. The normalized values of phases density have been plotted together with the real crystals which grew next to the centers of the specified electrodes and were determined by a metric measurement of their size. The measured size has varied in range $(0,4-0,9) \mathrm{mm}$. Next, the presence of crystals has been marked on the charts as the normalized density value 0 . The value 1 goes for the sucrose solution.

It has been confirmed that using the sensor (a), it is not possible to detect the regions of crystals. The relative electrical permittivity of the sucrose solution is too high in contrast to both the reactor's border and crystals to distinguish them. In the case of the sensor (b), the grown crystals are noticeable on the reconstructed image. Nevertheless, the paths of normalized density values on both charts express a significantly lower detection ability than for the sensor (c). The reconstructed normalized density values are expected to be close to 1 but the sensor (b) provides no higher than 0,83 and similarly for crystals at least 0,28 instead of 0 . Even if the image is optically correct, it does not provide valuable information for further analysis. The paths for the sensor (c) in most reliable way reflect the positions of the real crystals in terms of their size as well as the density. It is because the sensor (c) with the stabilized electrodes has a higher sensitivity in a border area than the sensor (b).

However, it is worth noting that in the case of ECT sensors, the measurement is performed with the entire electrode's surface, not one image point, as it was considered in the case of the charts. The ECT technique is not able to distinguish the singular crystals. The diagnostic information is averaged and produces the error in crystals' size reconstruction. This can be seen on the chart for 19-23 on the right side. Taking the above into consideration, the measurement resolution of 3D ECT allows detecting the localization of crystals (limescale). The application of stabilized electrodes improves the sensitivity of the sensor and provides the images better suitable for further analysis. However, we should keep in mind that other noninvasive techniques can penetrate the reactor's interior and distinguish single crystals (size, shape and position). But as

Figure 8 Three dimensional images of normalized sucrose crystals distribution, where the value of normalized density 1 means solution and 0 crystals

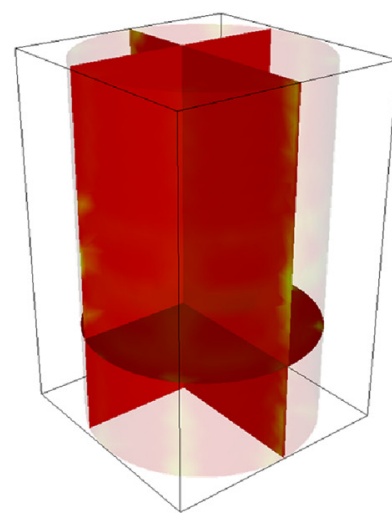

(a)

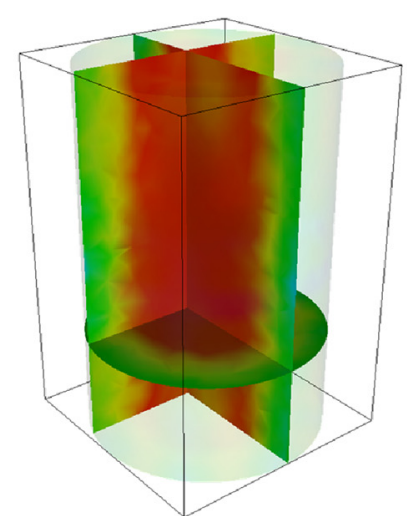

(b)

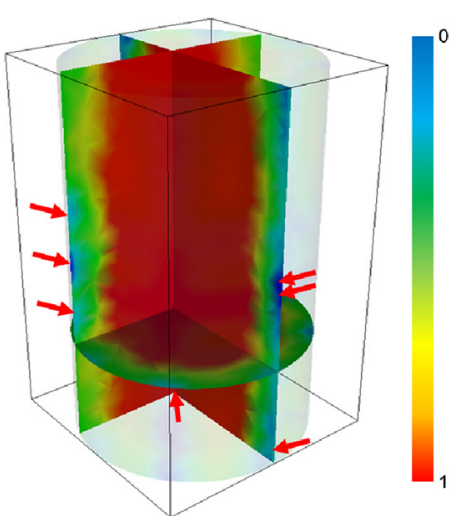

(c)

Figure 9 Normalized values of phases density in the crystallization reactor for three ECT sensors

Electrodes $9-13$

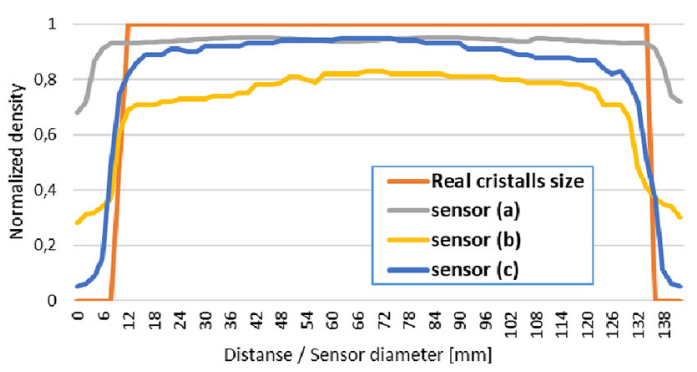

Electrodes $19-23$

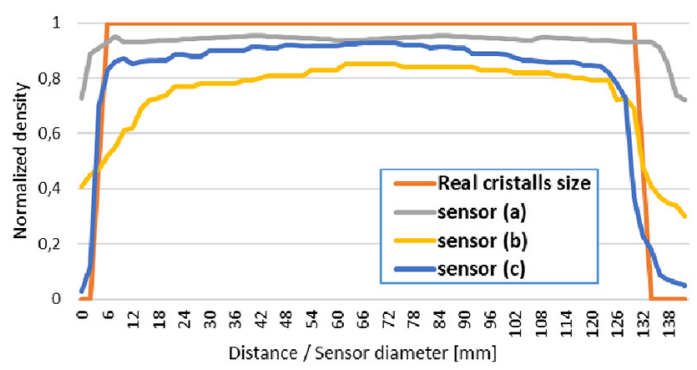


usual, the quality does not go hand in hand with the quantity. The high measurement frequency of electrical tomography systems recompenses the poor detection abilities. Therefore, they are still commonly applied in diagnostic systems of dynamic flow processes. Moreover, the ECT in comparison to the ERT is resistant to the electrodes' overgrowth with the crystals (limescale) as it happens for instance in the sucrose industry.

\section{Conclusions and future works}

In this work, the ability of the 3D ECT technique for crystallization process monitoring has been studied. The dedicated sensor construction has been proposed to increase its sensitivity in the border area, where the crystals grow. The 3D image reconstruction process for ECT has been described. Many aspects of computer models designing have been pointed out. The potential field distribution and behavior, as well as the sensitivities model of ECT sensor, have been analyzed. Finally, it has been shown that the ECT technique can be applied to the diagnosis of crystallization. The crystals distribution can be identified. Thanks to the electrical isolation, ECT is resistant to the crystals' overgrowth. Despite the benefits of applied stabilizing electrodes, some disadvantages must be listed. Note that in the new sensor construction, the total electrodes count is doubled. Additionally, the specificity of the measurement strategy imposes the specialist instrument usage. The multiswitching device, used in these experiments, is slow. Nevertheless, the crystallization processed by cooling in the laboratory reactor is luckily also slow. It was possible, therefore, to meet the diagnostic requirements and point the next research directions. That is why the future works will strongly consider the measurement instrument optimization (speed up) and the development of image analysis algorithms to identify crystallization parameters in the context of crystals concertation and pipeline's limescale evaluation in the industrial conditions.

\section{References}

Abbas, A., Nobbs, D. and Romagnoli, J.A. (2002), "Investigation of on-line optical particle characterization in reaction and cooling crystallization systems. Current state of the art", Measurement Science and Technology, Vol. 13 No. 3, pp. 349-356.

Al Hosani, E., Zhang, M. and Soleimani, M. (2015), "A limited region electrical capacitance tomography for detection of deposits in pipelines", IEEE Sensors fournal, Vol. 15 No. 11, pp. 6089-6099.

Banasiak, R., Wajman, R., Jaworski, T., Fiderek, P., Fidos, H., Nowakowski, J. and Sankowski, D. (2014), "Study on twophase flow regime visualization and identification using 3D electrical capacitance tomography and fuzzy-logic classification", International fournal of Multiphase Flow, Vol. 58, pp. 1-14.

Banasiak, R., Wajman, R., Jaworski, T., Fiderek, P., Kapusta, P. and Sankowski, D. (2017), "Two-phase flow regime three-dimensional visualization using electrical capacitance tomography - algorithms and software", Informatics Control Measurement in Economy and Environment Protection, Vol. 7 No. 1, pp. 11-16.
Banasiak, R., Wajman, R., Sankowski, D. and Soleimani, M. (2010), "Three-Dimensional nonlinear inversion of electrical capacitance tomography data using a complete sensor model", Progress in Electromagnetics Research, Vol. 100, pp. 219-234.

Barrett, P. Smith, B. Worlitschek, J. Bracken, V. O’Sullivan, B. and O'Grady, D. (2005), “A Review of the Use of Process Analytical Technology for the Understanding and Optimization of Production Batch Crystallization Processes", American Chemical Society, available at: https:// doi.org/10.1021/OP049783P

Besenhard, M.O., Hohl, R., Hodzic, A., Eder, R.J.P. and Khinast, J.G. (2014), "Modeling a seeded continuous crystallizer for the production of active pharmaceutical ingredients", Crystal Research and Technology, Vol. 49 Nos 2/3, pp. 92-108.

Bischof, J.C., Mahr, B., Choi, J.H., Behling, M. and Mewes, D. (2007), "Use of X-ray tomography to map crystalline and amorphous phases in frozen biomaterials", Annals of Biomedical Engineering, Vol. 35 No. 2, pp. 292-304.

Butler, M.F. Glaser, N. Weaver, A.C. Kirkland, M. and Heppenstall-Butler, M. (2006), "Calcium Carbonate Crystallization in the Presence of Biopolymers", American Chemical Society, available at: https://doi.org/10.1021/ CG050436W

Carlson, W.D. and Denison, C. (1992), "Mechanisms of porphyroblast crystallization: results from high-resolution computed X-ray tomography”, Science, Vol. 257 No. 5074, pp. 1236-1239.

Correa, C., Valero, C., Barreiro, P., Diago, M.P. and Tardaguila, J. (2012), "Feature extraction on vineyard by gustafson kessel FCM and K-means", 2012 16th IEEE Mediterranean Electrotechnical Conference, IEEE, pp. 481-484.

Cui, Z., Wang, Q., Xue, Q., Fan, W., Zhang, L., Cao, Z., Sun, B., et al. (2016), "A review on image reconstruction algorithms for electrical capacitance/resistance tomography", Sensor Review, Vol. 36 No. 4, pp. 429-445.

Fiderek, P., Kucharski, J. and Wajman, R. (2017), "Fuzzy inference for two-phase gas-liquid flow type evaluation based on raw 3D ECT measurement data", Flow Measurement and Instrumentation, Vol. 54, pp. 88-96.

Fujiwara, M., Nagy, Z.K., Chew, J.W. and Braatz, R.D. (2005), "First-principles and direct design approaches for the control of pharmaceutical crystallization", fournal of Process Control, Vol. 15 No. 5, pp. 493-504. Elsevier,

Gao, Z., Rohani, S., Gong, J. and Wang, J. (2017), "Recent developments in the crystallization process: toward the pharmaceutical industry", Engineering, Vol. 3 No. 3, pp. 343-353.

Garbaa, H., Jackowska-Strumiłło, L., Grudzień, K. and Romanowski, A. (2016), "Simulation of gravitational solids flow process and its parameters estimation by the use of electrical capacitance tomography and artificial neural networks", Informatics, Control, Measurement in Economy and Environment Protection, Vol. 6 No. 2, pp. 34-37.

Georgiou, D., Bendos, D., Kalis, M. and Koutis, C. (2018), "Removal and/or prevention of limescale in plumbing tubes by a radio-frequency alternating electric field inductance device", Fournal of Water Process Engineering, Vol. 22, pp. 34-40. 
Grudzien, K., Chaniecki, Z., Romanowski, A., Niedostatkiewicz, M. and Sankowski, D. (2012), "ECT image analysis methods for shear zone measurements during silo discharging process", Chinese fournal of Chemical Engineering, Vol. 20 No. 2, pp. 337-345.

Grudzien, K., Romanowski, A., Chaniecki, Z., Niedostatkiewicz, M. and Sankowski, D. (2010), "Description of the silo flow and bulk solid pulsation detection using ECT”, Flow Measurement and Instrumentation, Vol. 21 No. 3, pp. 198-206.

Grudzien, K., Chaniecki, Z., Romanowski, A., Sankowski, D., Nowakowski, J. and Niedostatkiewicz, M. (2016), "Application of twin-plane ECT sensor for identification of the internal imperfections inside concrete beams", 2016 IEEE International Instrumentation and Measurement Technology Conference Proceedings, IEEE, pp. 1-6.

Harada, Y., Yamamura, H., Ueshima, Y., Mizoguchi, T., Saito, N. and Nakashima, K. (2018), "Simultaneous evaluation of viscous and crystallization behaviors of silicate melts by capacitance and viscosity measurements", ISIF International, Vol. 58 No. 7, pp. 1285-1292.

Hierzenberger, P., Stifter, D., Leiss-Holzinger, E., Eder, G., D'Amore, A., Acierno, D. and Grassia, L. (2010), "In-situ monitoring of polymer crystallization by PolarizationSensitive optical coherence tomography", pp. 43-45.

Isaksen, Ø. (1996), "A review of reconstruction techniques for capacitance tomography”, Measurement Science and Technology, Vol. 7 No. 3, pp. 325-337.

Jha, S.K., Karthika, S. and Radhakrishnan, T.K. (2017), "Modelling and control of crystallization process", ResourceEfficient Technologies, Vol. 3 No. 1, pp. 94-100.

Kapusta, P., Majchrowicz, M., Sankowski, D. and Banasiak, R. (2012), "Application of GPU parallel computing for acceleration of finite element method based 3D reconstruction algorithms in electrical capacitance tomography", Image Processing \& Communications, Vol. 17 No. 4, pp. 339-346.

Kawabata, K. (2011), “Automatic detection of crystallization evaluation region by using image processing", Sensor Review, Vol. 31 No. 3, pp. 207-217.

Kowalska, A., Banasiak, R., Romanowski, A. and Sankowski, D. (2019), "3D-Printed multilayer sensor structure for electrical capacitance tomography", Sensors, , Vol. 19 No. 15 , pp. 3416.

Kryszyn, J., Smolik, W.T., Radzik, B., Olszewski, T. and Szabatin, R. (2014), "Switchless charge-discharge circuit for electrical capacitance tomography", Measurement Science and Technology, , Vol. 25 No. 11, p. 115009.

Kryszyn, J., Wróblewski, P., Stosio, M., Wanta, D., Olszewski, T. and Smolik, W.T. (2017), "Architecture of EVT4 data acquisition system for electrical capacitance tomography", Measurement, Vol. 101, pp. 28-39.

Li, M. Wilkinson, D. Patchigolla, K. Mougin, P. Roberts, K.J. and Tweedie, R. (2004), "On-Line Crystallization Process Parameter Measurements Using Ultrasonic Attenuation Spectroscopy", American Chemical Society, available at: https://doi.org/10.1021/CG030041H

Loser, T., Wajman, R. and Mewes, D. (2001), "Electrical capacitance tomography: image reconstruction along electrical field lines", Measurement Science and Technology, Vol. 12 No. 8, pp. 1083.

Mazurkiewicz, L., Banasiak, R., Wajman, R., Dyakowski, T. and Sankowski, D. (2005), "Towards 3D capacitance tomography", 4th World Congress in Industrial Process Tomography.

Mougin, P., Thomas, A., Wilkinson, D., White, G., Roberts, K.J., Herrmann, N., Jack, R. (2003), “On-line monitoring of a crystallization process”, AIChE fournal, Vol. 49 No. 2, pp. 373-378, doi: 10.1002/aic.690490209.

Mullin, J.W. (2001), Crystallization, Butterworth-Heinemann, available at: www.elsevier.com/books/crystallization/mullin/ 978-0-7506-4833-2 (accessed 23 July 2019).

Ohta, Y., Kitayama, M., Kaneko, K., Toh, S., Shimizu, F. and Morinaga, K. (2005), "In situ measurement of capacitance: a method for fabricating nanoglass", Fournal of the American Ceramic Society, Vol. 88 No. 6, pp. 1634-1636.

Panczyk, M. and Sikora, J. (2016), "A new imaging algorithm for electric capacitance tomography", Proceedings of Electrotechnical Institute, Vol. 63 No. 0, pp. 27-40.

Primrose, K.M. (2008), “Applications of electrical tomography to improve the performance of crystallization, precipitation and mixing processes", Fournal of the Southern African Institute of Mining and Metallurgy, Vol. 108, pp. 591-595.

Rashid, W.N.A., Mohamad, E.J., Rahim, R.A., Abdullah, J. and Ameran, H.L.M. (2016), "Electrical capacitance tomography: a review on portable ECT system and hardware design", Sensor Review, Vol. 36 No. 1, pp. 64-70.

Romanowski, A., Grudzień, K., Garbaa, H. and JackowskaStrumiłło, L. (2017), "Parametric methods for ECT inverse problem solution in solid flow monitoring", Informatics Control Measurement in Economy and Environment Protection, Vol. 7 No. 1, pp. 50-54.

Rózsa, L. (1996), “On-line monitoring of supersaturation in sugar crystallisation”, International Sugar fournal, Vol. 98, pp. 660-661.

Rózsa, L., Rózsa, J. and Kilpinen, S. (2016), “Crystal growth and crystallization control tactics in industrial sugar crystallizers part 1. Crystal growth", International Sugar fournal, Vol. 118, pp. 746-755.

Rymarczyk, T. (2016), "New methods to determine moisture areas by electrical impedance tomography", International Fournal of Applied Electromagnetics and Mechanics, Vol. 52 Nos 1/2, available at: https://doi.org/10.3233/JAE-162071.

Rymarczyk, T., Tchorzewski, P. and Sikora, J. (2017), "Coupling boundary element method with level set method to solve inverse problem", Informatics Control Measurement in Economy and Environment Protection, Vol. 7 No. 1, pp. 80-83.

Rymarczyk, T., Tchorzewski, P., Adamkiewicz, P., Duda, K., Szumowski, J. and Sikora, J. (2017), "Practical implementation of electrical tomography in a distributed system to examine the condition of objects", IEEE Sensors fournal, Vol. 17 No. 24, pp. 8166-8186.

Rzasa, M.R. (2009), "The measuring method for tests of horizontal two-phase gas-liquid flows, using optical and capacitance tomography", Nuclear Engineering and Design, Vol. 239 No. 4, pp. 699-707.

Rzasa, M.R., Wajman, R., Grudzien, K. and Romanowski, A. (2007), "The capacitive tomograph of higher sensitivity near 
the sensor wall", 5th World Congress in Industrial Process Tomography.

Shekunov, B.Y. and York, P. (2000), "Crystallization processes in pharmaceutical technology and drug delivery design", fournal of Crystal Growth, Vol. 211 Nos 1/4, pp. 122-136. Nos

Sikora, J. (2007), Boundary Element Method for Impedance and Optical Tomography, Oficyna Wydawnicza Politechniki Warszawskiej, Warszawa.

Smolik, W., Kryszyn, J., Olszewski, T. and Szabatin, R. (2017), "Methods of small capacitance measurement in electrical capacitance tomography", Informatics Control Measurement in Economy and Environment Protection, Vol. 7 No. 1, pp. 105-110.

Soleimani, M. (2006), "Three-dimensional electrical capacitance tomography imaging", Insight - Non-Destructive Testing and Condition Monitoring, Vol. 48 No. 10, pp. 613-617.

Temerk, Y.M., El-Abdein, O.Z., Abdullah, I.D. and Amin, S. T. (2014), "Sugar crystallization in low temperatures: the theory and practice by advanced sugar crystallization control program", International fournal of Engineering and Technical Research, Vol. 2 No. 6, pp. 41-48.

Villanueva, D., Posada, R., González, I., García, Á. and Martínez, A. (2015), "Monitoring of a sugar crystallization process with fuzzy logic and digital image processing", Fournal of Food Process Engineering, Vol. 38 No. 1, pp. 19-30.
Wang, F., Marashdeh, Q., Fan, L.-S. and Warsito, W. (2010), "Electrical capacitance volume tomography: design and applications", Sensors, Vol. 10 No. 3, pp. 1890-1917.

Winn, D. and Doherty, M.F. (2000), "Modeling crystal shapes of organic materials grown from solution", AIChE fournal, Vol. 46 No. 7, pp. 1348-1367.

Yang, W.Q. and Peng, L. (2003), "Image reconstruction algorithms for electrical capacitance tomography", Measurement Science and Technology, Vol. 14 No. 1, pp. R1-R13.

Zhao, Y. and Hammond, R.B. (2011), "Characterization of crystallisation processes with electrical impedance spectroscopy", Nuclear Engineering and Design, Vol. 241 No. 6, pp. 1938-1944.

\section{About the author}

Radosław Wajman, $\mathrm{DSc}, \mathrm{PhD}$, Eng. is an Professor at the Institute of Applied Computer Science at the Lodz University of Technology. In his work, he deals with the issues in the field of three-dimensional electrical capacitance tomography, image reconstruction and processing, fuzzy inference, software engineering for non-invasive monitoring, diagnosis and industrial automatic control system for multiphase flow processes. Radosław Wajman can be contacted at: radoslaw. wajman@p.lodz.pl 